\title{
"Double Trouble": Preoperative Embolization in a Case of Double Rupture of Hepatic Hemangioma during Pregnancy
}

\author{
Soumil Singhal ${ }^{1} \quad$ Mangerira Chinnappa Uthappa $^{1}$ \\ ${ }^{1}$ Department of Intervention Radiology and Intervention Oncology, \\ BGS Gleneagles Global Hospital, Bangalore, Karnataka, India
}

\begin{abstract}
Address for correspondence Soumil Singhal, MD, Department of Intervention Radiology and Intervention Oncology, BGS Gleneagles Global Hospital, Kengeri, Bangalore 560060, Karnataka, India (e-mail: drsoumilsinghal75@gmail.com).
\end{abstract}
Abstract
Keywords
- pregnancy
- cesarean section
- embolization
- hepatic hemangioma

Hepatic hemangiomas are the most common benign space-occupying lesion of the liver. These lesions are associated with the gestational age of the patient. They can easily be missed and can rarely present with complications. Spontaneous rupture of hemangioma is a rare occurrence. Preoperative arterial embolization of hemangiomas plays a vital role to reduce intraoperative bleed during surgical resection. Here is a case of a young pregnancy presenting with a complicated outcome.

\section{Introduction}

Hepatic hemangiomas are the most common benign space-occupying lesion of the liver. ${ }^{1}$ They are usually small and asymptomatic; however, they can develop a complication such as spontaneous rupture that can go completely unnoticed, especially in the background of a gravid uterus. Preoperative arterial embolization of hemangiomas plays a vital role to reduce intraoperative bleed during surgical resection. Here is a case with a similar clinical history and its multidisciplinary approach in the management of the complication.

\section{Case Presentation}

A 28-year-old patient in term gestation presented with an acute onset of abdominal pain following which she was immediately shifted to the closest health care facility. On clinical examination, the attending physician felt that pain was not because of ongoing labor following which ultrasound and blood workup were performed. Blood evaluation was suggestive of a hemoglobin drop (10-6 g\%), and portable ultrasound showed viable fetus; however, there was peritoneal free fluid with fluid showing echogenic internal content. A large focal lesion was seen in the left lobe of liver, which had a heterogeneous echotexture. Doppler was not performed because of the patient's clinical condition. The patient was prepared for an emergency cesarean section with exploratory laparotomy. A healthy live fetus was delivered. On further evaluation, the peritoneal cavity contained a hematoma, and the liver showed a large lesion with a focus of bleed. The bleeder was surgically managed by ligating the point of bleed. Three points were ligated. After 24 hours, the patient's condition deteriorated with a further drop in hemoglobin concentration and oxygen saturation. The patient was intubated and shifted to a tertiary health care setup for further management. Further imaging was performed at our center.

Emergency computed tomography (CT) of the abdomen was performed (16 slice, GE Healthcare, Milwaukee, Wisconsin, United States). The scan was performed in the supine position in suspended respiration. Omnipaque (Iohexol 350, General Electric Healthcare Little Chalfont, United Kingdom) was used as intravenous contrast media. The Contrast was injected using a pressure injector through an 18-gauge cannula sited in the left upper limb vein. Patients underwent a dual-phase enhanced CT scan with the arterial phase taken at 15 seconds and the venous phase taken at 60 seconds. Technical parameters were set as pitch (1.2), collimation (arterial: $16 \times 0.6 \mathrm{~mm}$ and venous: $24 \times 1.2 \mathrm{~mm}$ ), rotational time (0.6 seconds), $\mathrm{kV}$ and $\mathrm{mA}$ (120), and bolus trigger at descending aorta.
License terms

(ㅇ) (1) $\ominus \circledast$ 
Noncontrast CT showed enlarged left lobe with a large round lesion with areas of mixed density within, which was well demarcated from the liver and hyperdense intraperitoneal content suggestive of hematoma. On contrast-enhanced CT left lobe of the liver was enlarged and replaced with multiple lesions showing early arterial enhancement with contrast enhancement following the aortic enhancement pattern on successive phases ( - Fig. 1). The largest lesion was found to measure $13.5 \times 8 \times 6 \mathrm{~cm}$ $(\mathrm{AP} \times \mathrm{TR} \times \mathrm{CC})$ and showed peripheral nodular enhancement with central nonenhancing area suggestive of hemangioma (-Fig. 2). In view of the patient's clinical condition (postpartum day 2 with a low hemoglobin concentration), immediate surgery was deferred, and a two-step approach was contemplated by the surgical team. The first step was to perform a preoperative embolization and the second step to perform a left lobe resection after the patient's condition was stabilized.Preoperative embolization was performed under mild sedation. Through a right femoral arterial access, the celiac and hepatic arteries were cannulated, and the angiographic run was acquired. Superselectively catheterization of the left hepatic artery branch was performed with a $2.7 \mathrm{~F}$ microcatheter (Progreat, Terumo, Tokyo, Japan) (-Fig. 3), and suspicious foci of contrast staining was embolized to stasis using 300 to $500 \mu \mathrm{m}$ polyvinyl alcohol microspheres (Cook, Bloomington, IN, USA) (-Fig. 4). Further embolization was not contemplated as the patient was planned for surgery, and the embolization was performed to attain clinical stability. Postembolization angiography showed stasis of flow with no more staining. Post-embolization, there was no further drop in hemoglobin concentration and the value stabilized at $7.4 \mathrm{~g} \%$. Postprocedure a second-step left lobe
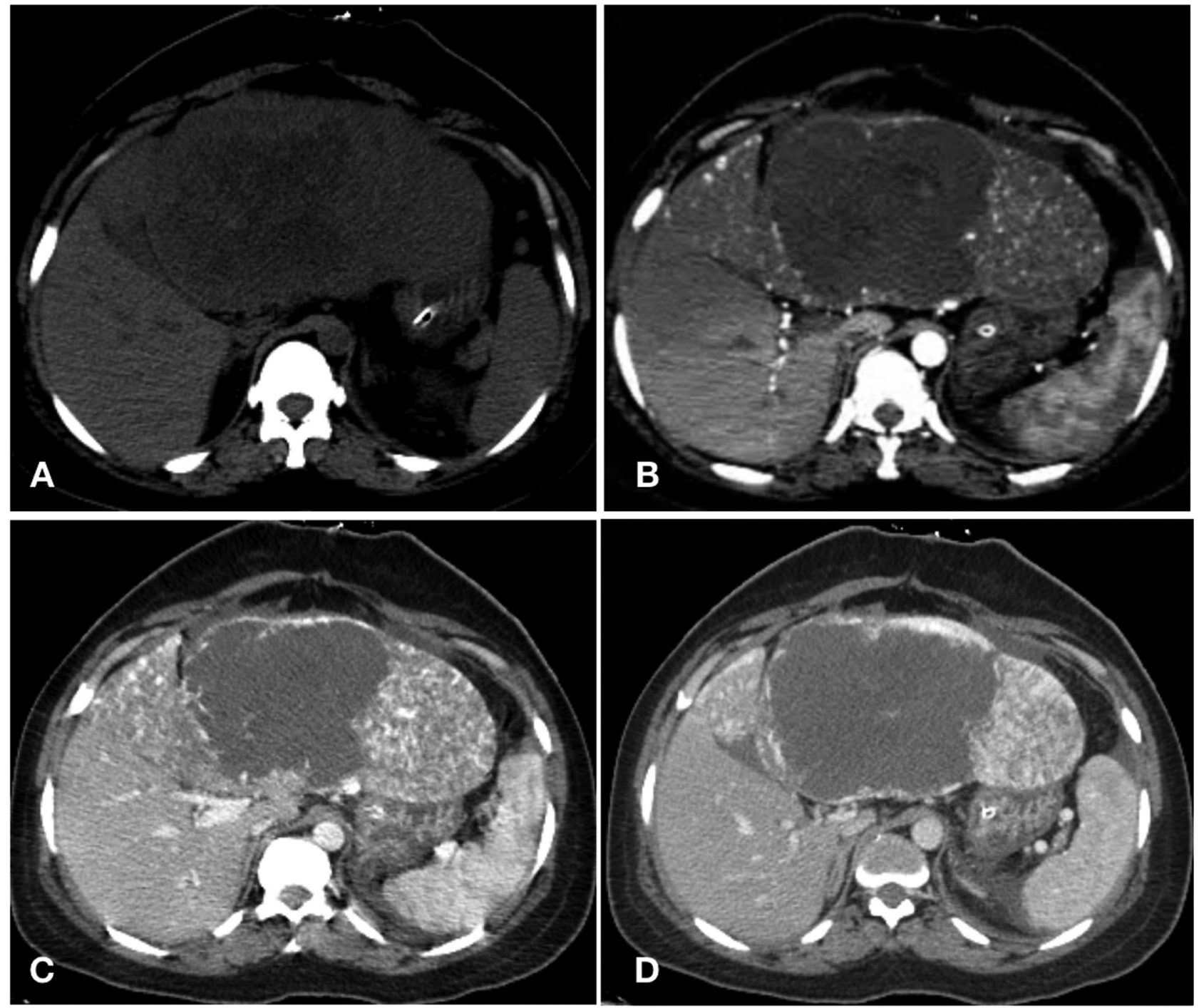

Fig. 1 (A-D) Axial images of contrast-enhanced CT of the abdomen. (A) Noncontrast scan shows bulky left lobe of liver with a large focal lesion showing some mixed density areas within. (B) Arterial phase shows multiple foci of intense arterial enhancement with a large lesion seen in segment 3. No active contrast extravasation was seen. (C, D) Portal and venous phase shows the lesion following the HU value corresponding to that of the aorta. The large segment 3 lesion shows peripheral puddling of contrast with the central nonenhanced area, which is suggestive of a hematoma. 


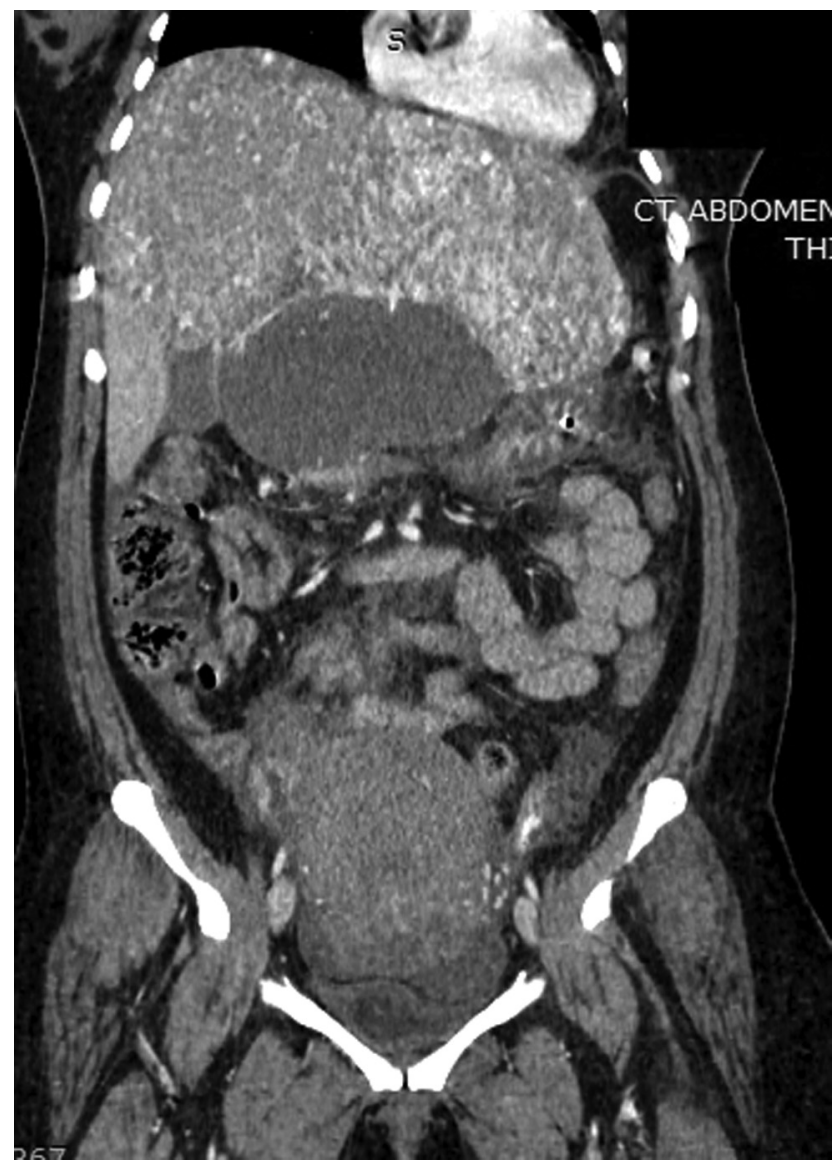

Fig. 2 Contrast-enhanced CT of the abdomen. Coronal reformat image shows multiple liver lesions with a segment 3 lesion showing peripheral contrast puddling. No active contrast extravasation or capsular breach was seen. resection was performed after 6 hours post-embolization. On the table, the surgeons found ease in resection due to the presurgical embolization as the blood loss was significantly low (30-40 mL). Post-surgery, the patient did well with no postoperative complication. Histopathologic evaluation of the specimen was suggestive of multiple capillary and cavernous hemangioma with many showing embolic particles within (-Fig. 5).

\section{Discussion}

Hepatic hemangiomas are the most common benign space-occupying lesion of the liver with an incidence rate of 0.4 to $7.3 \% .^{1,2}$ It is a vascular malformation of congenital origin with ectatic vessels that are either atypical or arranged irregularly. They can be either cavernous or capillary type. Lesions larger than $4 \mathrm{~cm}$ are known as a giant hemangioma. Hemangiomatosis that is, multiple hemangiomas are seen in up to $10 \%$ of cases. ${ }^{3}$ Hemangiomas are more common in females and have found to be more common during pregnancy and in patients taking exogenous estrogen or steroids. Hemangioma in pregnancy is different as its growth is triggered by various hormones., ${ }^{4,5}$ Lesion size is seen to be associated with the patient's gestational age. ${ }^{6}$ The progression and regression of the condition in the various group of patients have been poorly understood. In the vast majority of patients, the condition is asymptomatic and is incidentally detected on imaging. Giant hemangiomas can be symptomatic in $90 \%$ of cases. ${ }^{6}$ The patient presents with acute pain in the background of hemorrhage and thrombosis. Other complications include
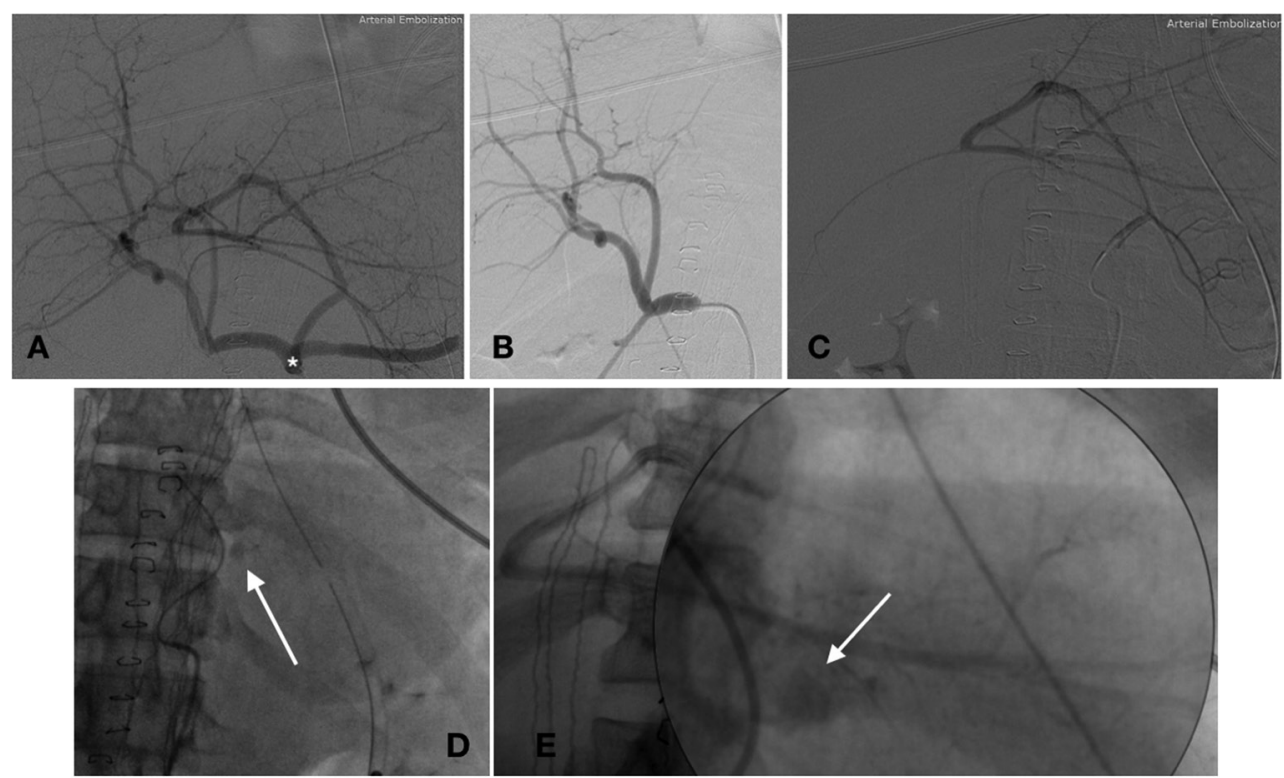

Fig. 3 (A-E) Conventional angiography. Celiac artery run showing replaced left hepatic artery, which is arising from the left gastric artery. (B) Common hepatic artery run. (C) Left hepatic run with the contrast blush. (D) Areas of contrast blush arising from the left hepatic artery vessels. (E) Magnified area of the contrast blush. 

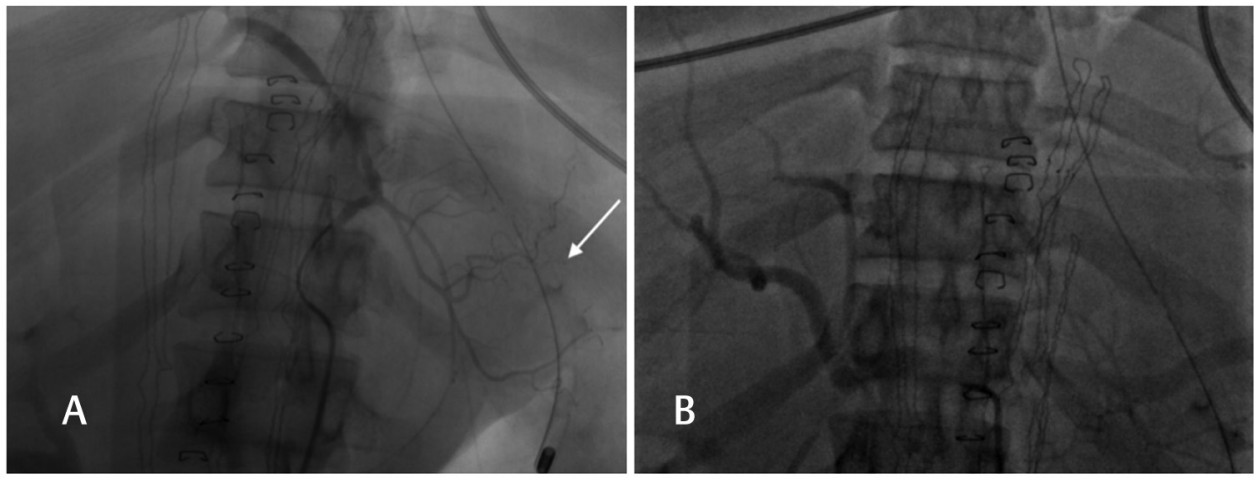

Fig. 4 (A, B) Postembolization images.
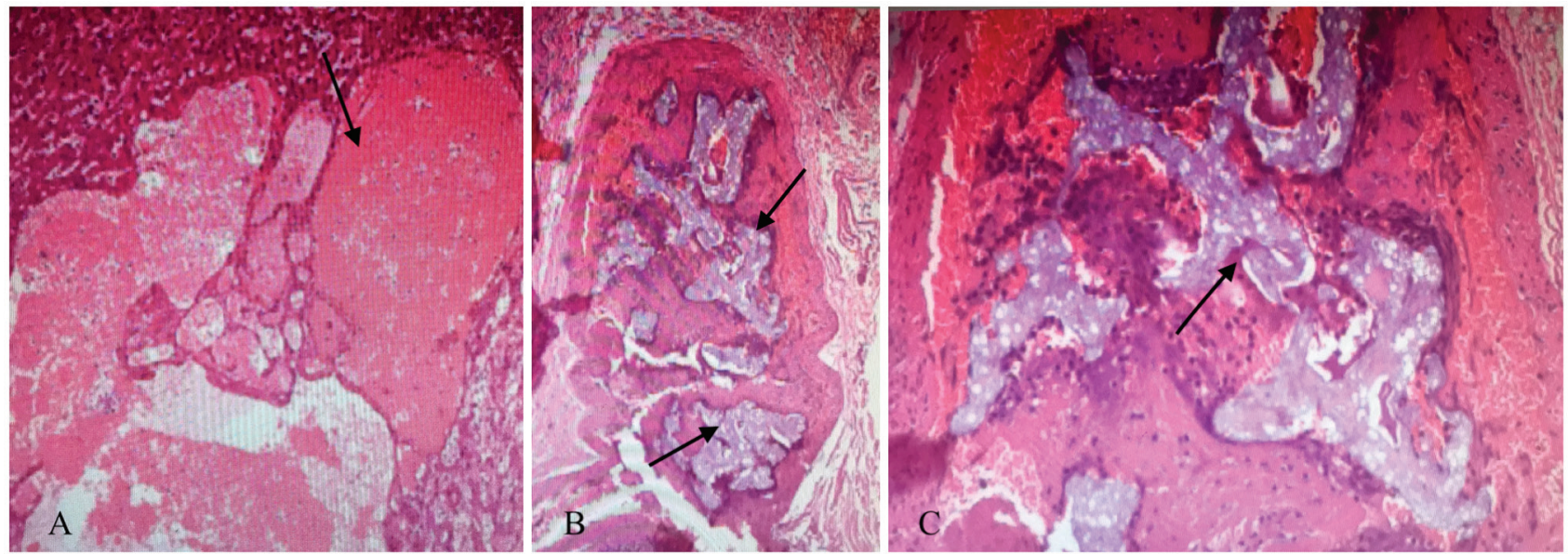

Fig. 5 (A-C) Histopathology of the resected liver specimen. (A) Large vascular spaces of varying sizes lined by flat endothelial cells, these spaces are separated by fibrous septa. $(\mathbf{B}, \mathbf{C})$ Few embolized spaces with the embolic material within the space.

anemia, thrombocytopenia, and hypofibrinogenemia. ${ }^{7}$ The lesion can easily be missed due to vague symptoms including epigastric pain, nausea, and vomiting. Spontaneous rupture is extremely rare and life threatening. Four types of rupture and bleeding have been described in the literature that includes (1) free rupture with hemoperitoneum, (2) intrahepatic hemorrhage, (3) double rupture, and (4) bleeding in the biliary tree. ${ }^{8}$ The hypothesis for spontaneous rupture is several and includes rupture secondary to the splitting of overlying liver parenchyma, necrosis secondary to rapid growth, and erosion of the hepatic vessels among a few causes. ${ }^{8}$

Various treatment option in treating asymptomatic hemangioma includes steroids, surgical resection, radiation therapy, and embolization. ${ }^{9}$ Worsening of symptoms, bleeding, or enlarging lesion size are indications for intervention. Various embolic agents can be used including PVA particles, gel foam slurry, iso-butyl cyanoacrylate, and coils mainly. ${ }^{10}$ Because of its permanent embolic nature, PVA particle was considered as an embolic of choice in our case. ${ }^{9-11} \mathrm{~A}$ review of the literature has revealed only a few cases of pregnancy associated with a complicated hemangioma. ${ }^{5.11-17}$ Transarterial embolization is a valid management tool due to evolving consumables. Presurgical embolization has shown very good outcome. ${ }^{18,19}$ Postembolic complication includes abdominal distention, fever, nausea, vomiting, nontargeted embolization, hepatic dysfunction, and rarely bile duct injury. However, transarterial embolization is a very safe and effective option in patients with high risk for surgery.

\section{Conclusion}

This case offers several learning points: (1) Hemangiomas are common during pregnancy and presents with a vague atypical presentation, (2) difficulty in diagnosis during pregnancy and the limitation of imaging, and (3) treatment management of the condition depends on the patient's clinical status.

\section{Key Points}

1. Spontaneous double rupture of giant hemangioma during pregnancy is a rare occurrence that requires a multidisciplinary approach in the management of the condition.

2. Transarterial embolization is a safe, effective, minimally invasive procedure with a good outcome, especially in complicated hemangiomas.

3. Presurgical transarterial embolization is a very effective tool in the armamentarium of the interventional radiologist. 


\section{Conflict of Interest}

None declared.

\section{References}

1 Unal E, Francis F, Aquino A, Xu R, Morgan G, Teperman L. Liver transplant for mixed capillary-cavernous hemangioma masquerading as hepatocellular carcinoma in a patient with hepatocellular carcinoma. Exp Clin Transplant 2011;9(5):344-348

2 John TG, Greig JD, Crosbie JL, Miles WF, Garden OJ. Superior staging of liver tumors with laparoscopy and laparoscopic ultrasound. Ann Surg 1994;220(6):711-719

3 Langkowski JH. [A ruptured cavernous hemangioma of the liver] [in German]. RoFo Fortschr Geb Rontgenstr Nuklearmed 1990;153(4):473-475

4 Glinkova V, Shevah O, Boaz M, Levine A, Shirin H. Hepatic haemangiomas: possible association with female sex hormones. Gut 2004;53(9):1352-1355

5 Saegusa T, Ito K, Oba N, et al. Enlargement of multiple cavernous hemangioma of the liver in association with pregnancy. Intern Med 1995;34(3):207-211

$6 \mathrm{Au}$ WY, Liu CL. Growth of giant hepatic hemangioma after triplet pregnancy. J Hepatol 2005;42(5):781

7 Ishak KG, Rabin L. Benign tumors of the liver. Med Clin North Am 1975;59(4):995-1013

8 Donati M, Stavrou GA, Donati A, Oldhafer KJ. The risk of spontaneous rupture of liver hemangiomas: a critical review of the literature. J Hepatobiliary Pancreat Sci 2011;18(6):797-805

9 Gaur P, Umredkar AB, Anand A, Rathod J, Agrawal M. Role of hepatic artery embolization in giant hemangioma of liver. Int J Sci Stud 2017;5(1):40-49
10 Sun $\mathrm{JH}$, Nie $\mathrm{CH}$, Zhang $\mathrm{YL}$, et al. Transcatheter arterial embolization alone for giant hepatic hemangioma. PLoS One 2015;10(8):e0135158

11 Mohan S, Gupta A, Verma A, Kathura MK, Baijal SS. Case report: non-surgical management of a giant liver hemangioma. Indian J Radiol Imaging 2007;17:81-83

12 Rubin I. Larger pedunculated cavernous angioma of the liver reaching down into the pelvis and causing obstetric difficulty. Am J Obstet Dis Women Child 1918;77:273-276

13 Sewell JH, Weiss K. Spontaneous rupture of hemangioma of the liver. A review of the literature and presentation of illustrative case. Arch Surg 1961;83:729-733

14 Creasy GW, Flickinger F, Kraus RE. Maternal liver hemangioma in pregnancy as an incidental finding. Obstet Gynecol 1985;66(3, Suppl):10S-13S

15 Marques R, Taborda F, Jorge CS, Areias J, Rodrigues AM. Successful outcome in a pregnancy complicated by large hepatic hemangioma. Acta Obstet Gynecol Scand 1997;76(6):606-607

16 Krasuski P, Poniecka A, Gal E, Wali A. Intrapartum spontaneous rupture of liver hemangioma. J Matern Fetal Med 2001;10(4):290-292

17 Ebina Y, Hazama R, Nishimoto M, et al. Resection of giant liver hemangioma in a pregnant woman with coagulopathy: case report and literature review. J Prenat Med 2011;5(4):93-96

18 Jain V, Ramachandran V, Garg R, Pal S, Gamanagatti SR, Srivastava DN. Spontaneous rupture of a giant hepatic hemangioma-sequential management with transcatheter arterial embolization and resection. Saudi J Gastroenterol 2010;16(2):116-119

19 Vassiou K, Rountas H, Liakou P, Arvanitis D, Fezoulidis I, Tepetes K. Embolization of a giant hepatic hemangioma prior to urgent liver resection. Case report and review of the literature. Cardiovasc Intervent Radiol 2007;30(4):800-802 